\title{
Número mínimo de medições para a avaliação acurada de características agronômicas de pinhão-manso
}

\author{
Paulo Eduardo Teodoro(1), Rhayanne Dias Costa ${ }^{(2)}$, Rodrigo Barros Rocha ${ }^{(3)}$ e Bruno Galvêas Laviola(2)
}

(1)Universidade Estadual de Mato Grosso do Sul, Unidade Universitária de Aquidauana, Rodovia Aquidauana, Km 12, Zona Rural, CEP 79200-000 Aquidauna, MS, Brasil. E-mail: eduteodoro@hotmail.com (2)Embrapa Agroenergia, Parque Estação Biológica (PqEB), W3 Norte (Final), CEP 70770-901 Brasília, DF, Brasil. E-mail: rhdico@gmail.com, bruno.laviola@embrapa.br ${ }^{(3)}$ Embrapa Rondônia, Rodovia BR-364, Km 5,5, Zona Rural, Caixa Postal 127, CEP 76815-800 Porto Velho, RO, Brasil. E-mail: rodrigo.rocha@embrapa.br

Resumo - O objetivo deste trabalho foi identificar o método mais eficaz para estimar coeficientes de repetibilidade em progênies de meios-irmãos de pinhão-manso (Jatropha curcas) e predizer o número mínimo de medições necessárias para algumas características agronômicas da oleaginosa. Avaliaram-se 18 famílias de meios-irmãos, em delineamento de blocos ao acaso, com três repetições, de 2009 a 2013. Nesse período, as seguintes características foram avaliadas: altura de plantas, diâmetro do caule, número de ramos por planta, projeção da copa na linha, projeção da copa na entrelinha e produtividade de grãos. O coeficiente de repetibilidade ( $\mathrm{r}$ ) foi estimado a partir de diferentes estratégias: análise de variância; análise dos componentes principais (ACP), com base na matriz de correlações (CPCOR); ACP, com base na matriz de variâncias e covariâncias fenotípicas (CPCOV); e análise estrutural, com base na matriz de correlações. A estratégia CPCOV proporciona estimativas mais acuradas do coeficiente de repetibilidade e do número de medições necessárias (no mínimo quatro) para avaliação acurada (mínimo 80\%) das características, devido ao comportamento cíclico das progênies.

Termos para indexação: Jatropha curcas, análise multivariada, eficiência de seleção, precisão experimental, repetibilidade.

\section{Minimum number of measurements for the accurate evaluation of agronomic traits in physic nut}

\begin{abstract}
The objective of this work was to identify the most effective method to estimate the coefficient of repeatability in progenies of half-sib families of physic nut (Jatropha curcas), and to predict the minimum number of measurements required for some agronomic traits of this oilseed crop. Eighteen half-sib families, in a randomized complete block design with three replicates, were evaluated from 2009 to 2013. In this period, the following traits were evaluated: plant height, stem diameter, number of branches per plant, crown projection on the line, crown projection on the space between rows, and grain yield. The repeatability coefficient (r) was estimated considering different strategies: analysis of variance; principal component analysis (PCA) based on the correlation matrix (PCCOR); PCA based on the matrix of phenotypic variance and covariance (PCCOV); and structural analysis based on the correlation matrix. The PCCOV strategy provides the most accurate estimates of the repeatability coefficient and of the number of measurements (minimum of four), which are required for an accurate evaluation (minimum $80 \%$ accuracy) of the traits, due to the cyclical behavior of the progenies.
\end{abstract}

Index terms: Jatropha curcas, multivariate analysis, selection efficiency, experimental precision, repeatability.

\section{Introdução}

O pinhão-manso (Jatropha curcas L.) é uma espécie oleaginosa perene da família Euphorbiaceae, com bom potencial produtivo de grãos e de óleo com alta qualidade para a produção de biodiesel. Nativa da América Central (Fairless, 2007), essa espécie foi trazida para o Brasil para ser utilizada como cerca viva e para produção de óleo em pequena escala (Rosado et al., 2010). Atualmente, o pinhão-manso vem sendo pesquisado para a produção de óleo em larga escala, com vistas à produção de biodiesel e bioquerosene (Durães et al., 2011).

Em pesquisas com genótipos de pinhão-manso, características como a altura de plantas, o diâmetro do caule, o número de ramos por planta, a projeção 
da copa na linha, a projeção da copa na entrelinha e a produtividade de grãos são comumente avaliadas (Laviola et al., 2012). Nessas pesquisas, a correta discriminação dos genótipos depende de sua avaliação em condições e intensidades que permitam precisão (menor dispersão) e acurácia (pequeno viés entre a estimativa e o parâmetro) das estimativas. Portanto, o dimensionamento adequado do número de medições a serem realizadas é fundamental para a caracterização dos genótipos, pois, vários anos podem ser necessários para quantificar a expressão de uma característica que se manifesta ao longo do tempo.

Recentemente, as pesquisas de campo com pinhãomanso têm-se intensificado, principalmente quanto a: desempenho agronômico (Everson et al., 2013; Laviola et al., 2014); estimativas de parâmetros genéticos (Laviola et al., 2012; Wani et al., 2012; Brasileiro et al., 2013); divergência genética (Laviola et al., 2010; Alves et al., 2013; Shabanimofrad et al., 2013; Sharma et al., 2013); e análise de trilha (Das et al., 2010; Spinelli et al., 2010). Para identificar indivíduos com desempenho superior, normalmente, têm-se realizado entre uma e quatro medições, ao longo do ciclo da cultura.

Durante o processo de seleção de genótipos com vistas ao lançamento de cultivares ou à escolha de parentais para recombinação, é importante que se tenha certeza da superioridade genética dos indivíduos. Para tanto, muitas vezes são necessárias medições repetidas do mesmo indivíduo (Negreiros et al., 2008). Assim, o dimensionamento do número de medições a serem realizadas, por meio do coeficiente de repetibilidade, é fundamental para o melhoramento de espécies perenes, pois fornece uma aproximação do valor máximo que pode ter a herdabilidade de uma característica no sentido amplo (Cruz et al., 2004). Além disso, esta informação também é importante por possibilitar a determinação de qual é o menor ciclo de seleção possível, o que tem reflexo direto sobre os gastos com recursos humanos e financeiros da pesquisa.

$\mathrm{O}$ dimensionamento do número de medições vem sendo realizado para diversas culturas perenes, como a aceroleira (Lopes et al., 2001), goiabeira (Degenhardt et al., 2002), araçazeiro e pitangeira (Danner et al., 2010), pessegueiro (Bruna et al., 2012), laranjeira-doce (Negreiros et al., 2014) e bananeira (Lessa et al., 2014). Em geral, os autores destas pesquisas observaram que métodos multivariados são mais precisos em vez do método univariado; porém, é necessário que pesquisas com outras culturas sejam feitas antes da generalização destes resultados.

O objetivo deste trabalho foi identificar o método mais eficaz para estimar coeficientes de repetibilidade em progênies de meios-irmãos de pinhão-manso (Jatropha curcas) e predizer o número mínimo de medições necessárias para algumas características agronômicas da oleaginosa.

\section{Material e Métodos}

O experimento foi realizado na área experimental da Embrapa Cerrados, de 2009 a 2013, situada em Planaltina, DF, a $15^{\circ} 35^{\prime} 30^{\prime \prime} \mathrm{S}, 47^{\circ} 42^{\prime} 30^{\prime \prime} \mathrm{W}$ e a $1.007 \mathrm{~m}$ de altitude. O clima é tropical, com inverno seco e verão chuvoso (Aw), segundo a classificação de Köppen-Geiger, com temperatura média anual de $21^{\circ} \mathrm{C}$, umidade relativa de $68 \%$ e precipitação pluvial média anual de $1.100 \mathrm{~mm}$.

Avaliaram-se 18 famílias de meios-irmãos - 801, $802,803,804,805,806,807,808,809,810,811,812$, $813,814,815,816,817$ e $818-$, em delineamento de blocos ao acaso, com três repetições e cinco plantas dispostas em linha, por parcela útil. Detalhes sobre a genealogia das progênies se encontram em Laviola et al. (2014).

$\mathrm{O}$ ensaio foi implantado com mudas de aproximadamente 60 dias de cultivo, no espaçamento de $4 \times 2 \mathrm{~m}$. As práticas de manejo basearam-se em Dias et al. (2007), com adaptações de acordo com os resultados de pesquisa com pinhão-manso no Brasil e no mundo (Bahadur et al., 2013; Carels et al., 2012; Resende et al., 2013).

Quatro medições foram realizadas quanto aos seguintes caracteres: altura de plantas (AP, $\mathrm{m})$; diâmetro do caule (DC, cm); número de ramos por planta (NRP); projeção da copa na linha (PCL, m); projeção da copa na entrelinha (PCE, $\mathrm{m}$ ); e produtividade de grãos $\left(\mathrm{kg} \mathrm{ha}^{-1}\right)$. Os caracteres AP, DC, NRP, PCL e PCE foram mensurados aos $6,18,30$ e 42 meses, e a produtividade foi avaliada aos 30, 42, 54 e 66 meses após o plantio. Para estimar o coeficiente de repetibilidade (r) com maior consistência, avaliaram-se quatro métodos: análise de variância (Anova); análise dos componentes principais (ACP), com base na matriz de correlações (CPCOR); ACP, com base na matriz de variâncias e covariâncias fenotípicas (CPCOV); e análise estrutural, com base na matriz de correlações ( $r$ médio, AECOR). 
Inicialmente, utilizou-se o teste de Mauchly (1940), para verificar se as estruturas das matrizes de covariância atendiam à condição de esfericidade. Aplicou-se o teste de Lilliefors (Sprent \& Smeeton, 2007) para verificar o pressuposto de normalidade dos erros e, para avaliar a aleatoriedade dos erros, utilizouse o teste de sequência (run test) (Storck et al., 2011). Empregou-se o teste de Bartlett (Sprent \& Smeeton, 2007) para verificar a homogeneidade das variâncias dos erros, entre as progênies. A aditividade do modelo matemático foi verificada pelo teste de aditividade de Tukey (Snedecor \& Cochran, 1989). As hipóteses das pressuposições (normalidade, aleatoriedade, homogeneidade e aditividade) foram testadas a $5 \%$ de probabilidade. As análises estatísticas foram realizadas com auxílio dos programas SAS (SAS Institute, Cary, NC, EUA) e R (R Foundation for Statistical Computing, Viena, Áustria).

Posteriormente, os dados foram submetidos à Anova, em que o modelo estatístico adotado considerou dois fatores de variação (Cruz et al., 2004): $Y_{i j}=\mu+g_{i}+a_{j}+\varepsilon_{i j}$, em que: $Y_{i j}$ é a observação referente à i-ésima progênie de pinhão-manso, na j-ésima medição; $\mu$ é a média geral; $g_{i}$ é o efeito aleatório da i-ésima progênie, sob a influência do ambiente permanente; $a_{j}$ é o efeito fixo do ambiente temporário, na j-ésima medição

$$
\sum_{j=1}^{n} a_{j}=0
$$

e $\varepsilon_{\mathrm{ij}}$ é o erro experimental estabelecido pelos efeitos temporários do ambiente, na j-ésima medição da i-ésima progênie. As médias foram comparadas pelo teste de Scott e Knott a 5\% de probabilidade.

Com base na Anova, foram obtidas as estimativas dos quadrados médios - das medições $\left(\mathrm{QM}_{\mathrm{M}}\right)$, dos genótipos $\left(\mathrm{QM}_{\mathrm{G}}\right)$ e do resíduo $\left(\mathrm{QM}_{\mathrm{R}}\right)$-, bem como o valor do teste $\mathrm{F}$ para genótipo $\left(\mathrm{Fc}=\mathrm{QM}_{\mathrm{G}} / \mathrm{QM}_{\mathrm{R}}\right)$. Em seguida, estimou-se o coeficiente de repetibilidade pela equação,

$$
\mathrm{R}=\left\{\mathrm{QM}_{\mathrm{G}}-\mathrm{QM}_{\mathrm{R}} /\left[\mathrm{QM}_{\mathrm{G}}+(\eta-1) \mathrm{QM}_{\mathrm{R}}\right]\right\},
$$

em que: $\mathrm{QM}_{\mathrm{G}}$ é o quadrado médio do genótipo; QMR é o quadrado médio do resíduo; e $\eta$ é o número de medições realizadas.

O método CPCOR consiste na obtenção de uma matriz de correlação entre as progênies, em cada par de medição. São determinados, na matriz, os autovalores e os respectivos autovetores normalizados associados. $\mathrm{O}$ autovetor cujos elementos apresentam mesmo sinal e magnitudes próximas é aquele que expressa a tendência dos clones de manter, ao longo dos anos, suas posições relativas em relação aos demais (Abeywardena, 1972; Cruz et al., 2004). Seu estimador é dado por,

$$
\mathrm{r}=\bar{\lambda}_{1}-1 / \eta-1,
$$

em que $\widehat{\lambda}_{1}$ é o autovalor da matriz de covariância $(\widehat{\Gamma})$ ou da matriz de correlação $(\widehat{\Gamma})$.

Para o método dos componentes principais CPCOV, o estimador da repetibilidade é dado por:

$$
\mathrm{r}=\hat{\lambda}_{1}-\hat{\sigma}_{\mathrm{Y}}^{2} / \widehat{\sigma}_{\mathrm{Y}}^{2}-(\eta-1),
$$

em que $\widehat{\sigma}_{Y}^{2}$ é a variância fenotípica (Cruz et al., 2004).

A obtenção do coeficiente de repetibilidade mediante o método AECOR apresenta apenas diferenças conceituais em relação aos métodos baseados nos componentes principais. Mansour et al. (1981), autores deste método, consideram $\mathrm{R}$ a matriz paramétrica de correlações entre tratamentos em cada par de avaliações, e $\widehat{R}$, o seu estimador. A obtenção do coeficiente de repetibilidade com base no AECOR é feita mediante a utilização da equação,

$$
\mathrm{r}=\widehat{\alpha}^{\prime} \hat{\mathrm{R}} \hat{\alpha}-1 / \eta-1,
$$

em que $\widehat{\alpha}$ é o autovetor associado ao maior autovalor de $\hat{\mathrm{R}}$.

Para cada caráter, determinou-se o número mínimo de medições necessárias para predizer o valor real dos indivíduos $(\eta)$, com base em um coeficiente de determinação $\left(\mathrm{R}^{2}\right)$ pré-estabelecido $(80$ e $85 \%)$ que, conforme Cruz et al. (2004), é dado por,

$$
\mathrm{n}=\mathrm{R}^{2}(1-\mathrm{r}) /\left(1-\mathrm{R}^{2}\right) \mathrm{r},
$$

em que r é o coeficiente de repetibilidade, obtido de acordo com um dos diferentes métodos utilizados.

$\mathrm{O}$ coeficiente de determinação genotípica $\left(\mathrm{R}^{2}\right)$, que representa a percentagem de certeza da predição do valor real das progênies, com base em $\eta$ medições, foi obtido pela expressão $\mathrm{R}^{2}=\eta \times \mathrm{r} / 1+\mathrm{r}(\eta-\mathrm{r})$. As análises estatísticas foram realizadas com auxílio dos programas Genes (Cruz, 2013) e Microsoft Excel.

\section{Resultados e Discussão}

Verificou-se, para todas as características analisadas, que: as estruturas das variâncias atenderam à condição de esfericidade, conforme o teste de Mauchly (1940); os erros ajustaram-se à distribuição normal, de acordo 
com o teste de Lilliefors (Sprent \& Smeeton, 2007); as variâncias residuais foram homogêneas, conforme o teste de Bartlett (Sprent \& Smeeton, 2007); os erros foram aleatórios, segundo o teste de sequência; e houve aditividade do modelo matemático pelo teste de Tukey (Snedecor \& Cochran, 1989) (Tabela 1). A condição de esfericidade da matriz de covariância indica que a análise de variância univariada pode ser aplicada ao modelo de blocos ao acaso, em esquema de parcelas subdivididas no tempo. $\mathrm{O}$ atendimento das pressuposições - aleatoriedade, normalidade dos erros, aditividade do modelo matemático e homogeneidade das variâncias - é importante para a manutenção das taxas de erro tipo I e tipo II dos testes de hipótese utilizados neste trabalho (Storck et al., 2011). O teste F mostrou existência de variabilidade genética $(p<0,01)$ entre as progênies de pinhão-manso, em todos os caracteres avaliados, o que é indicativo de que há a possibilidade de seleção de genótipos superiores.

Embora a produtividade seja uma das características mais importantes para a seleção de acessos superiores de pinhão-manso (Laviola et al., 2013), também é necessário avaliar outros caracteres agronômicos para estimar ganhos indiretos com a seleção que favoreçam ou dificultem o desenvolvimento de novas variedades. A AP, por exemplo, é um caráter importante na escolha do arranjo espacial mais apropriado para plantio (Dias et al., 2007), em que as progênies $801,803,804,805$, $806,807,808,810,811,812,813,814,815,816$ e 817 merecem destaque por terem apresentado as maiores médias (Tabela 2).

Para o caráter DC, as progênies $801,803,804,806$, $807,808,812,813,814,815,816,817$ e 818 obtiveram as maiores médias, o que indica que elas apresentam maior capacidade de sustentação da planta e, portanto, menor suscetibilidade ao tombamento (Dias et al., 2007). As progênies 804, 806, 807, 808, 809, 810, 811, $812,813,814,816,817$ e 808 apresentaram maior NRP. De acordo com Spinelli et al. (2010), este caráter é importante para a cultura do pinhão-manso, em razão de sua relação direta com a produtividade de grãos e o rendimento de óleo.

Spinelli et al. (2010) verificaram que maiores valores de PCL e PCE proporcionam maior volume da copa de pinhão-manso, que é um caráter importante e vem sendo estudado recentemente com o objetivo de se desenvolver práticas de poda mais apropriadas para o cultivo da oleaginosa. Os autores também constataram que plantas com maior PCE apresentaram maior produtividade de grãos, teor e rendimento de óleo. Assim, as progênies 801,813 e 814 se destacaram por apresentar as maiores médias de PCL e PCE.

As progênies 801, 803, 804, 806, 807, 808, $809,811,812$ e 813 obtiveram as maiores médias de produtividade, durante as quatro medições. O desempenho produtivo desses genótipos apresentou magnitude superior à verificada por Spinelli et al. (2014), que avaliaram 16 progênies de meios-irmãos em Porto Velho (RO), por três medições.

Tabela 1. Resumo da análise de variância e valores-p para os testes de Mauchly, para a esfericidade da matriz de covariâncias; de Tukey, para a aditividade do modelo matemático; de sequências, para aleatoriedade dos erros; de Bartlett, para a homogeneidade de variâncias residuais; e de Lilliefors, para a normalidade dos erros, na avaliação de seis caracteres, em 18 progênies de pinhão-manso (Jatropha curcas), durante quatro medições.

\begin{tabular}{lccccccc}
\hline Fonte de variação & GL & \multicolumn{9}{c}{ Quadrados médios } \\
\cline { 3 - 8 } & & AP & DC & NRP & PCL & PCE & Produtividade \\
\hline Medições & 3 & $17,49^{*}$ & $228,36^{*}$ & $991,72^{*}$ & $7,38^{*}$ & $11,25^{*}$ & $6.449 .626^{*}$ \\
Genótipo & 17 & $0,06^{*}$ & $1,25^{*}$ & $3,53^{*}$ & $0,03^{*}$ & $0,06^{*}$ & $145.981^{*}$ \\
\hline Resíduo & 51 & 0,01 & 0,24 & 0,90 & 0,01 & 0,01 & 53.114 \\
\hline & & & \multicolumn{5}{c}{ Valor de p } \\
Esfericidade & - & 0,08 & 0,07 & 0,20 & 0,25 & 0,19 & 0,07 \\
Aditividade & - & 0,09 & 0,09 & 0,41 & 0,28 & 0,34 & 0,21 \\
Aleatoriedade & - & 0,32 & 0,337 & 0,19 & 0,92 & 0,85 & 0,35 \\
Homogeneidade & - & 0,08 & 0,08 & 0,08 & 0,47 & 0,22 & 0,80 \\
Normalidade & - & 0,06 & 0,07 & 0,10 & 0,58 & 0,19 & 0,17
\end{tabular}

*Significativo pelo teste F, a 1\% de probabilidade. AP, altura de plantas; DC, diâmetro do caule; NRP, número de ramos por planta; PCL, projeção da copa na linha; e PCE, projeção da copa na entrelinha. 
A média dos caracteres avaliados aumentou ao longo das três primeiras medições e estabilizouse entre a terceira e a quarta medição, indício de que há comportamento cíclico das progênies de pinhão-manso. Isto é importante, pois, quando um estudo de repetibilidade é realizado com genótipos ainda não estabilizados, estimativas menos precisas podem ser encontradas, o que poderia levar o pesquisador a acreditar que o aumento do número de medições é a solução para o problema. Em alguns casos, a ausência de medição em fases iniciais, em que não há manifestação completa do potencial genético do material estudado, pode aumentar a estimativa de $r$ (Laviola et al., 2013).

As estimativas de $\mathrm{r}$ pelo método da Anova foram inferiores às observadas com os demais métodos, independentemente do caráter avaliado (Tabela 3).

Tabela 2. Valores médios dos caracteres avaliados em 18 progênies de meios-irmãos de pinhão-manso (Jatropha curcas), em quatro medições ${ }^{(1)}$.

\begin{tabular}{lcccccc}
\hline Progênie & AP & $\begin{array}{c}\text { PCL } \\
\text {-------------(m)------------ }\end{array}$ & $\begin{array}{c}\text { PCE } \\
(\mathrm{cm})\end{array}$ & $\begin{array}{c}\text { NRP } \\
\text { Produtividade } \\
\left(\mathrm{kg} \mathrm{ha}^{-1}\right)\end{array}$ \\
\hline 801 & $2,15 \mathrm{a}$ & $1,24 \mathrm{a}$ & $1,47 \mathrm{a}$ & $10,58 \mathrm{a}$ & $7,77 \mathrm{~b}$ & $1.291,88 \mathrm{a}$ \\
802 & $1,69 \mathrm{c}$ & $0,94 \mathrm{~b}$ & $1,02 \mathrm{c}$ & $8,23 \mathrm{~b}$ & $7,88 \mathrm{~b}$ & $817.62 \mathrm{~b}$ \\
803 & $2,10 \mathrm{a}$ & $1,02 \mathrm{~b}$ & $1,12 \mathrm{c}$ & $9,64 \mathrm{a}$ & $7,35 \mathrm{~b}$ & $1.270,42 \mathrm{a}$ \\
804 & $2,12 \mathrm{a}$ & $0,99 \mathrm{~b}$ & $1,13 \mathrm{c}$ & $9,57 \mathrm{a}$ & $8,35 \mathrm{a}$ & $1.109,63 \mathrm{a}$ \\
805 & $2,10 \mathrm{a}$ & $0,94 \mathrm{~b}$ & $1,01 \mathrm{c}$ & $8,95 \mathrm{~b}$ & $7,37 \mathrm{~b}$ & $894,90 \mathrm{~b}$ \\
806 & $2,14 \mathrm{a}$ & $0,98 \mathrm{~b}$ & $1,16 \mathrm{c}$ & $9,61 \mathrm{a}$ & $8,78 \mathrm{a}$ & $1.259,97 \mathrm{a}$ \\
807 & $2,09 \mathrm{a}$ & $1,09 \mathrm{a}$ & $1,13 \mathrm{c}$ & $9,84 \mathrm{a}$ & $9,03 \mathrm{a}$ & $1.118,91 \mathrm{a}$ \\
808 & $2,16 \mathrm{a}$ & $1,00 \mathrm{~b}$ & $1,23 \mathrm{~b}$ & $9,63 \mathrm{a}$ & $9,17 \mathrm{a}$ & $1.066,05 \mathrm{a}$ \\
809 & $1,96 \mathrm{~b}$ & $1,04 \mathrm{~b}$ & $1,20 \mathrm{c}$ & $9,09 \mathrm{~b}$ & $8,55 \mathrm{a}$ & $1.088,77 \mathrm{a}$ \\
810 & $2,15 \mathrm{a}$ & $0,94 \mathrm{~b}$ & $1,12 \mathrm{c}$ & $9,03 \mathrm{~b}$ & $8,47 \mathrm{a}$ & $1.012,59 \mathrm{~b}$ \\
811 & $2,03 \mathrm{a}$ & $1,06 \mathrm{~b}$ & $1,15 \mathrm{c}$ & $8,90 \mathrm{~b}$ & $8,28 \mathrm{a}$ & $1.136,36 \mathrm{a}$ \\
812 & $2,16 \mathrm{a}$ & $1,03 \mathrm{~b}$ & $1,17 \mathrm{c}$ & $9,69 \mathrm{a}$ & $8,72 \mathrm{a}$ & $1.257,80 \mathrm{a}$ \\
813 & $2,13 \mathrm{a}$ & $1,20 \mathrm{a}$ & $1,37 \mathrm{a}$ & $9,55 \mathrm{a}$ & $9,75 \mathrm{a}$ & $1.095,84 \mathrm{a}$ \\
814 & $2,17 \mathrm{a}$ & $1,18 \mathrm{a}$ & $1,38 \mathrm{a}$ & $10,21 \mathrm{a}$ & $9,12 \mathrm{a}$ & $1.001,02 \mathrm{~b}$ \\
815 & $2,21 \mathrm{a}$ & $1,11 \mathrm{a}$ & $1,25 \mathrm{~b}$ & $10,12 \mathrm{a}$ & $5,77 \mathrm{c}$ & $967,99 \mathrm{~b}$ \\
816 & $2,03 \mathrm{a}$ & $1,13 \mathrm{a}$ & $1,27 \mathrm{~b}$ & $9,78 \mathrm{a}$ & $8,28 \mathrm{a}$ & $914,56 \mathrm{~b}$ \\
817 & $2,18 \mathrm{a}$ & $1,06 \mathrm{~b}$ & $1,19 \mathrm{c}$ & $9,83 \mathrm{a}$ & $8,40 \mathrm{a}$ & $951,05 \mathrm{~b}$ \\
818 & $1,91 \mathrm{~b}$ & $1,17 \mathrm{a}$ & $1,27 \mathrm{~b}$ & $10,04 \mathrm{a}$ & $9,67 \mathrm{a}$ & $510,82 \mathrm{c}$ \\
\hline Mediç̃ão 1 & $0,65 \mathrm{c}$ & $0,41 \mathrm{~b}$ & $0,41 \mathrm{c}$ & $4,68 \mathrm{~b}$ & $0,70 \mathrm{c}$ & $417,89 \mathrm{c}$ \\
Medicção 2 & $2,19 \mathrm{~b}$ & $0,64 \mathrm{~b}$ & $0,66 \mathrm{~b}$ & $9,57 \mathrm{a}$ & $3,65 \mathrm{~b}$ & $644,94 \mathrm{~b}$ \\
Medição 3 & $2,70 \mathrm{a}$ & $1,43 \mathrm{a}$ & $1,68 \mathrm{a}$ & $10,98 \mathrm{a}$ & $12,65 \mathrm{a}$ & $1.508,75 \mathrm{a}$ \\
Medição 4 & $2,78 \mathrm{a}$ & $1,76 \mathrm{a}$ & $2,05 \mathrm{a}$ & $13,05 \mathrm{a}$ & $16,48 \mathrm{a}$ & $1.598,70 \mathrm{a}$ \\
\hline
\end{tabular}

${ }^{(1)}$ Médias seguidas de letras iguais não diferem pelo teste de Scott-Knott, a $5 \%$ de probabilidade. AP, altura de plantas; DC, diâmetro do caule; NRP, número de ramos por planta; PCL, projeção da copa na linha; e PCE, projeção da copa na entrelinha.
As estimativas de $\mathrm{r}$ com o uso de componentes principais (CPCOR e CPCOV) foram as maiores, o que resultou em menor número de medições necessárias para identificar genótipos de pinhão-manso superiores (Tabela 4). Esse resultado, possivelmente, decorreu do comportamento cíclico observado, ou seja, do aumento dos valores de cada caráter ao longo das medições (Cruz et al., 2004).

Quando o fator medições é significativo, as medidas podem ser afetadas por mudanças de ordem fisiológica regulares, irregulares ou sistemáticas. Como esse efeito pode variar de maneira e intensidade diferentes, entre as progênies de pinhão-manso, o método da Anova não permite isolar esse fator, que tem seus efeitos misturados ao erro experimental; isto pode levar à subestimação da repetibilidade e superestimação do número de medições necessárias (Cruz et al., 2004). Nesse caso, o método dos componentes principais, que leva em consideração o comportamento cíclico do

Tabela 3. Estimativas do coeficiente de repetibilidade (r) e de determinação $\left(\mathrm{R}^{2}\right)$, para as características avaliadas em 18 progênies de meios-irmãos de pinhão-manso (Jatropha curcas), em quatro medições, de acordo com as seguintes estratégias: Anova, análise de variância; CPCOR, componentes principais, com base na matriz de correlações; CPCOV, componentes principais, com base na matriz de variâncias e covariâncias fenotípicas; e AECOR, análise estrutural, com base na matriz de correlações ( $\mathrm{r}$ médio).

\begin{tabular}{lcccc}
\hline Parâmetro & Anova & CPCOR & CPCOV & AECOR \\
\hline \multicolumn{5}{c}{ Altura de plantas } \\
$\mathrm{r}$ & 0,63 & 0,63 & 0,73 & 0,63 \\
$\mathrm{R}^{2}$ & 0,87 & 0,87 & 0,91 & 0,89 \\
\hline \multicolumn{5}{c}{ Diâmetro do caule } \\
$\mathrm{r}$ & 0,51 & 0,63 & 0,55 & 0,62 \\
$\mathrm{R}^{2}$ & 0,81 & 0,87 & 0,83 & 0,87 \\
\hline \multicolumn{5}{c}{ Número de ramos por planta } \\
$\mathrm{r}$ & 0,42 & 0,81 & 0,48 & 0,46 \\
$\mathrm{R}^{2}$ & 0,75 & 0,95 & 0,78 & 0,77 \\
\hline $\mathrm{r}$ & 0,57 & 0,58 & 0,78 \\
$\mathrm{R}^{2}$ & 0,84 & 0,84 & 0,93 & 0,57 \\
\hline $\mathrm{r}$ & 0,57 & 0,59 & 0,79 & 0,89 \\
$\mathrm{R}^{2}$ & 0,84 & 0,85 & 0,94 & 0,57 \\
\hline \multicolumn{5}{c}{ Projeção da copa nas entrelinhas } \\
$\mathrm{r}$ & 0,30 & 0,52 & 0,57 \\
$\mathrm{R}^{2}$ & 0,64 & 0,82 & 0,84 & 0,51 \\
\hline
\end{tabular}


caráter, é o mais recomendado para estimar o $\mathrm{r}$ com acurácia. Isto ocorre porque, com esta metodologia, o autovetor é aquele que expressa a tendência das progênies de manter suas posições relativas nos períodos de medição (Abeywardena, 1972).

Resultados similares foram relatados sobre outras culturas que também expressaram comportamento cíclico, como a aceroleira (Lopes et al., 2001), goiabeira (Degenhardt et al., 2002), araçazeiro e pitangeira (Danner et al., 2010), pessegueiro (Bruna et al., 2012), laranjeira-doce (Negreiros et al., 2014) e bananeira (Lessa et al., 2014), em que o método da Anova superestimou as estimativas de $r$ e superestimou o número de medições necessárias, em comparação aos métodos baseados nos componentes principais (CPCOR e CPCOV). Assim, em razão dos resultados obtidos e de sua coerência com pesquisas publicadas, recomenda-se a utilização dos métodos baseados nos componentes principais (CPCOR e CPCOV) em trabalhos futuros.

Tabela 4. Estimativas do número de medições associadas a diferentes coeficientes de determinação $\left(\mathrm{R}^{2}\right)$, para as características avaliadas em 18 progênies de meios-irmãos de pinhão-manso (Jatropha curcas), em quatro medições, de acordo com as seguintes estratégias: Anova, análise de variância; $\mathrm{CPCOR}$, componentes principais, com base na matriz de correlações; CPCOV, componentes principais, com base na matriz de variâncias e covariâncias fenotípicas; e AECOR, análise estrutural, com base na matriz de correlações ( $\mathrm{r}$ médio).

\begin{tabular}{|c|c|c|c|c|}
\hline $\mathrm{R}^{2}$ & Anova & CPCOR & CPCOV & AECOR \\
\hline & \multicolumn{4}{|c|}{ Altura de plantas } \\
\hline 0,80 & 2 & 2 & 2 & 2 \\
\hline \multirow[t]{2}{*}{$\underline{0,85}$} & 3 & 3 & 2 & 3 \\
\hline & \multicolumn{4}{|c|}{ Diâmetro do caule } \\
\hline 0,80 & 4 & 2 & 3 & 2 \\
\hline \multirow[t]{2}{*}{$\underline{0,85}$} & 5 & 3 & 4 & 3 \\
\hline & \multicolumn{4}{|c|}{ Número de ramos por planta } \\
\hline 0,80 & 5 & 4 & 1 & 5 \\
\hline \multirow[t]{2}{*}{$\underline{0,85}$} & 8 & 6 & 2 & 7 \\
\hline & \multicolumn{4}{|c|}{ Projeção da copa nas linhas } \\
\hline 0,80 & 3 & 3 & 1 & 4 \\
\hline \multirow[t]{2}{*}{0,85} & 4 & 4 & 2 & 5 \\
\hline & \multicolumn{4}{|c|}{ Projeção da copa nas entrelinhas } \\
\hline 0,80 & 3 & 2 & 1 & 3 \\
\hline \multirow[t]{2}{*}{0,85} & 4 & 3 & 2 & 4 \\
\hline & \multicolumn{4}{|c|}{ Produtividade } \\
\hline 0,80 & 9 & 4 & 3 & 4 \\
\hline 0,85 & 12 & 5 & 4 & 5 \\
\hline
\end{tabular}

Com exceção das estimativas de $\mathrm{r}$ e $\mathrm{R}^{2}$ pelo método da Anova, para o caráter produtividade, os valores de $\mathrm{r}$ e $\mathrm{R}^{2}$ obtidos neste trabalho foram superiores aos limites confiáveis - $r \geq 0,40, R^{2} \geq 0,75$ - propostos por Bergo et al. (2013) e Oliveira \& Moura (2010), respectivamente. Além disto, os valores de $\mathrm{r}$ obtidos denotam boa capacidade das progênies de repetir a expressão de um caráter ao longo das medições, enquanto as estimativas de $\mathrm{R}^{2}$ encontradas expressam segurança na superioridade genética das progênies de pinhão-manso selecionadas.

Em todos os métodos de análise, verificou-se que AP, DC, DCL e DCE demandam menor número de medições do que as demais características (Tabela 4), indício de que apresentam maior regularidade dos indivíduos de uma medição para outra (Cruz et al., 2004). Portanto, essas característica podem ser selecionadas mais precocemente, em programas de melhoramento genético de pinhão-manso.

Para um coeficiente de determinação mínimo de $80 \%$, NRP e PROD demandaram maior número de medições, o que indica maior efeito do ambiente sobre estas características, ou seja, maior variância ambiental em relação à variância genética entre as progênies. De acordo com Cruz et al. (2004), características quantitativas como a produtividade são determinados por um conjunto de diferentes genes, que podem ser expressos em maior ou menor grau conforme a fase de desenvolvimento dos genótipos, o que pode "inflacionar" o número mínimo de medições para identificação genótipos superiores quanto a esta característica.

\section{Conclusões}

1. A metodologia da análise de componentes principais, com uso da matriz de variâncias e covariâncias fenotípicas, proporciona estimativas mais acuradas do coeficiente de repetibilidade e do número de medições necessárias para avaliação acurada das características estudadas, em razão do comportamento cíclico das progênies de pinhão-manso (Jatropha curcas).

2. Quatro medições, no mínimo, são necessárias para predizer o valor real das progênies de pinhão-manso, com acurácia mínima de $80 \%$ pelo método dos componentes principais, com base na matriz de variâncias e covariâncias fenotípicas. 


\section{Referências}

ABEYWARDENA, V. An application of principal component analysis in genetics. Journal of Genetics, v.61, p.27-51, 1972. DOI: 10.1007/BF02984099.

ALVES, A.A.; BHERING, L.L.; ROSADO, T.B.; LAVIOLA, B.G.; FORMIGHIERI, E.F.; CRUZ, C.D. Joint analysis of phenotypic and molecular diversity provides new insights on the genetic variability of the Brazilian physic nut germplasm bank. Genetics and Molecular Biology, v.36, p.371-381, 2013. DOI: 10.1590/ S1415-47572013005000033.

BAHADUR, B.; SUJATHA, M.; CARELS, N. (Ed.). Jatropha, challenges for a new energy crop: genetic improvement and biotechnology. New York: Springer Verlag, 2013. v.2, 614p. DOI: 10.1007/978-1-4614-4915-7.

BERGO, C.L.; NEGREIROS, J.R.S.; MIQUELONI, D.P.; LUNZ, A.M.P. Estimativas de repetibilidade de caracteres de produção em pupunheiras para palmito da raça Putumayo. Revista Brasileira de Fruticultura, v.35, p.829-836, 2013. DOI: 10.1590/ S0100-29452013000300020.

BRASILEIRO, B.P.; SILVA, S.A.; SOUZA, D.R.; SANTOS, P.A.; OLIVEIRA, R.S.; LYRA, D.H. Genetic diversity and selection gain in the physic nut (Jatropha curcas). Genetics and Molecular Research, v.12, p.2341-2350, 2013. DOI: 10.4238/2013. January.4.12.

BRUNA, E.D.; MORETO, A.L.; DALBÓ, M.A. Uso do coeficiente de repetibilidade na seleção de clones de pessegueiro para o Litoral Sul de Santa Catariana. Revista Brasileira de Fruticultura, v.34, p.206-215, 2012. DOI: 10.1590/S0100-29452012000100028.

CARELS, N.; SUJATHA, M.; BAHADUR, B. (Ed.). Jatropha, challenges for a new energy crop: farming, economics and biofuel. New York: Springer Verlag, 2012. v. 1, 599p. DOI: 10.1007/978-1-4614-4806-8.

CRUZ, C.D. GENES: a software package for analysis in experimental statistics and quantitative genetics. Acta Scientiarum. Agronomy, v.35, p.271-276, 2013. DOI: 10.4025/ actasciagron.v35i3.21251.

CRUZ, C.D.; REGAZZI, A.J.; CARNEIRO, P.C.S. Modelos biométricos aplicados ao melhoramento genético. 3.ed. Viçosa: Ed. da UFV, 2004. 480p.

DANNER, M.A.; RASEIRA, M. do C.B.; SASSO, S.A.Z.; CITADIN, I.; SCARIOT, S. Repetibilidade de caracteres de fruto em araçazeiro e pitangueira. Ciência Rural, v.40, p.2086-2091, 2010. DOI: $10.1590 / \mathrm{S} 0103-84782010005000163$.

DAS, S.; MISRA, R.C.; MAHAPATRA, A.K.; GATAYAT, B.P; PATTNAIK, R.K. Genetic variability, character association and path analysis in Jatropha curcas. World Applied Sciences Journal, v.8, p.1304-1308, 2010.

DEGENHARDT, J.; DUCROQUET, J.P.; REIS, M.S.; GUERRA, M.P.; NODARI, R.O. Efeito de anos e determinação do coeficiente de repetibilidade de características de frutos de goiabeira-serrana. Pesquisa Agropecuária Brasileira, v.37, p.1285-1293, 2002. DOI: 10.1590/S0100-204X2002000900012.
DIAS, L.A. dos S.; LEME, L.P.; LAVIOLA, B.G.; PALLINI , A.; PEREIRA, O.L.; DIAS, D.C.F.S. CARVALHO, M.; MANFIO, C.E.; SANTOS, A.S. dos; SOUSA, L.C.A. de; OLIVEIRA, T.S. de; PRETTI, L.A. Cultivo de pinhão manso (Jatropha curcas L.) para produção de óleo combustível. Viçosa:L. A. S. Dias, 2007. 40p.

DURÃES, F.O.M.; LAVIOLA, B.G.; ALVES, A.A. Potential and challenges in making physic nut (Jatropha curcas L.) a viable biofuel crop: the Brazilian perspective. CAB Reviews: Perspectives in Agriculture, Veterinary Science, Nutrition and Natural Resources, v.6, p. 1-8, 2011. DOI: 10.1079/ PAVSNNR20116043.

EVERSON, C.S.; MENGISTU, M.G.; GUSH, M.B. A field assessment of the agronomic performance and water use of Jatropha curcas in South Africa. Biomass and Bioenergy, v.59, p.59-69, 2013. DOI: 10.1016/j.biombioe.2012.03.013.

FAIRLESS, D. Biofuel: the little shrub that could - maybe. Nature, v.449, p.652-655, 2007. DOI: 10.1038/449652a.

LAVIOLA, B.G.; ALVES, A.A.; GURGEL, F. de L.; ROSADO, T.B.; ROCHA, R.B.; ALBRECHT, J.C. Estimates of genetic parameters for physic nut traits based in the germplasm two years evaluation. Ciência Rural, v.42, p.429-435, 2012. DOI: 10.1590/ S0103-84782012000300008

LAVIOLA, B.G.; OLIVEIRA, A.M.C. e.; BHERING, L.L.; ALVES, A.A.; ROCHA, R.B.; GOMES, B.E.L.; CRUZ, C.D. Estimates of repeatability coefficients and selection gains in Jatropha indicate that higher cumulative genetic gains can be obtained by relaxing the degree of certainty in predicting the best families. Industrial Crops and Products, v.51, p.70-76, 2013. DOI: 10.1016/j.indcrop.2013.08.016.

LAVIOLA, B.G.; ROSADO, T.B.; BHERING, L.L.; KOBAYASHI, A.K.; RESENDE, M.D.V. de. Genetic parameters and variability in physic nut accessions during early developmental stages. Pesquisa Agropecuária Brasileira, v.45, p.1117-1123, 2010. DOI: 10.1590/ S0100-204X2010001000010.

LAVIOLA, B.G.; SILVA, S.D. dos A. e; JUHÁSZ, A.C.P.; ROCHA, R.B.; OLIVEIRA, R.J.P. de; ALBRECHT, J.C.; ALVES, A.A.; ROSADO, T.B. Desempenho agronômico e ganho genético pela seleção de pinhão-manso em três regiões do Brasil. Pesquisa Agropecuária Brasileira, v.49, p.356-363, 2014. DOI: 10.1590/ S0100-204X2014000500005.

LESSA, L.S.; LEDO, C.A. da S.; AMORIN, E.P.; SILVA, S. de O. e. Estimativas de repetibilidade de híbridos diploides (AA) de bananeira. Pesquisa Agropecuária Brasileira, v.49, p.109-117, 2014. DOI: 10.1590/S0100-204X2014000200005.

LOPES, R.; BRUCKNER, C.H.; CRUZ, C.D.; LOPES, M.T.G.; FREITAS, G.B. de. Repetibilidade de características do fruto de aceroleira. Pesquisa Agropecuária Brasileira, v.36, p.507-513, 2001. DOI: 10.1590/S0100-204X2001000300015.

MANSOUR, H.; NORDHEIM, E.V.; RUTLEDGE, J.J. Estimators of repeatability. Theoretical and Applied Genetics, v.60, p.151-156, 1981. DOI: 10.1007/BF00264520.

MAUCHLY, J.W. Significance test for sphericity of a normal $\mathrm{n}$-variate distribution. The Annals of Mathematical Statistics, v.11, p.204-209, 1940. 
NEGREIROS, J.R. da S.; ANDRADE NETO, R. de C.; MIQUELONI, D.P.; LESSA, L.S. Estimativa de repetibilidade para caracteres de qualidade de frutos de laranjeira-doce. Pesquisa Agropecuária Brasileira, v.49, p.40-48, 2014. DOI: 10.1590/ S0100-204X2014000100006.

NEGREIROS, J.R. da S.; SARAIVA, L.L.; OLIVEIRA, T.K. de; ÁLVARES, V. de S.; RONCATTO, G. Estimativas de repetibilidade de caracteres de produção em laranjeiras-doces no Acre. Pesquisa Agropecuária Brasileira, v.43, p.1763-1768, 2008. DOI: 10.1590/ S0100-204X2008001200017.

OLIVEIRA, M. do S.P. de; MOURA, E.F. Repetibilidade e número mínimo de medições para caracteres de cacho de bacabi (Oenocarpus mapora). Revista Brasileira de Fruticultura, v.32, p.1173-1179, 2010. DOI: 10.1590/S0100-29452010005000120.

RESENDE, J.C.F. de; LONDE, L.N.; NEVES, W. dos S. Pinhão-manso. Nova Porteirinha: Epamig, 2013. 524p.

ROSADO, T.B.; LAVIOLA, B.G.; FARIA, D.A.; PAPPAS, M.R.; BHERING, L.L.; QUIRINO, B.; GRATTAPAGLIA, D. Molecular markers reveal limited genetic diversity in a large germplasm collection of the biofuel crop Jatropha curcas L. in Brazil. Crop Science, v.50, p.2372-2382, 2010. DOI: 10.2135/ cropsci2010.02.0112.

SHABANIMOFRAD, M.; RAFII, M.Y.; WAHAB, P.E.M.; BIABANI, A.R.; LATIF, M.A. Phenotypic, genotypic and genetic divergence found in 48 newly collected Malaysian accessions of Jatropha curcas L. Industrial Crops and Products, v.42, p.543-551, 2013. DOI: 10.1016/j.indcrop.2012.06.023.
SHARMA, H.K.; SHUKLA, A.; KUMAR, A.; SHUKLA, A.; CHOUDHARY, S.B.; JATOTHU, J.L. Variability and genetic diversity assessment in physic nut (Jatropha curcas L.). Journal of Medicinal Plants Research, v.7, p.2380-2391, 2013.DOI: 10.5897/JMPR12.1164.

SNEDECOR, G.W.; COCHRAN, W.G. Statistical methods. 8.ed. Ames: Iowa State University, 1989. 503p.

SPINELLI, V.M.; DIAS, L.A. dos S.; ROCHA, R.B.; RESENDE, M.D.V. Yield performance of half-sib families of physic nut (Jatropha curcas L.). Crop Breeding and Applied Biotechnology, v.14, p.49-53, 2014. DOI: 10.1590/S1984-70332014000100008.

SPINELLI, V.M.; ROCHA, R.B.;RAMALHO,A.R.;MARCOLAN, A.L.; VIEIRA JÚNIOR, J.R.; FERNANDES, C. de F.; MILITÃO, J.S.L.T.; DIAS, L.A. dos S. Componentes primários e secundários do rendimento de óleo de pinhão-manso. Ciência Rural, v.40, p.1752-1758, 2010. DOI: 10.1590/S0103-84782010005000129.

SPRENT, P.; SMEETON, N.C. Applied nonparametric statistical methods. 4.ed. Boca Raton: Chapman \& Hall, 2007. 530p. (Texts in Statistical Science).

STORCK, L.; RIBEIRO, N.D.; CARGNELUTTI FILHO, A. Precisão experimental de ensaios de feijão analisada pelo método de Papadakis. Pesquisa Agropecuária Brasileira, v.46, p.798-804, 2011. DOI: 10.1590/S0100-204X2011000800003.

WANI, T.A.; KITCHLU, S.; RAN, G. Genetic variability studies for morphological and qualitative attributes among Jatropha curcas L. accessions grown under subtropical conditions of North India. South African Journal of Botany, v.79, p.102-105, 2012. DOI: 10.1016/j.sajb.2011.10.009. 